Coherent and Incoherent Cutoff Frequency

The classic coherent imaging system uses a normally incident monochromatic plane wave for illumination.

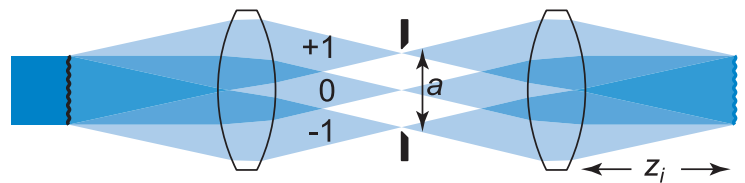

When the object is a grating with a single frequency at the coherent cutoff, the +1 and -1 orders are just barely accepted by the aperture of the imaging system. These three beams then produce a fringe pattern at the cutoff frequency. If the grating frequency is any greater than the cutoff frequency, then only the zero-order diffracted beam is passed by the imaging system, and the modulation is reduced to zero.

$$
f_{\text {cutorf }}^{\text {coherent }}=\frac{a / 2}{\lambda z_{i}}=\frac{1}{2 \lambda(F / \#)}=\frac{N A}{\lambda}
$$

In an incoherent imaging system, the grating is illuminated from every direction. Although frequencies just beyond the coherent cutoff are still blocked for a normally incident wavefront, the zero order and either the $+1^{\text {st }}$ or $-1^{\text {st }}$ orders can be passed by other angles of illumination and allowed to interfere at the image plane. The off-axis light continues to produce modulation until only one of the three beams can be passed; this occurs at twice the coherent cutoff.

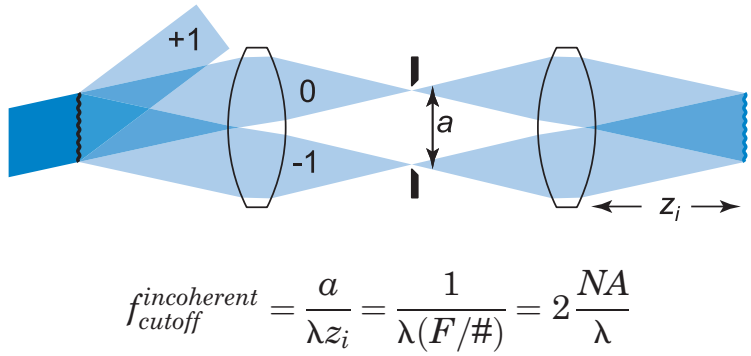

$\overline{\text { Field Guide to Physical Optics }}$ 


\section{Rayleigh Criterion}

The Rayleigh criterion is a common metric for determining whether two points or lines are resolved. It states that the first zeros of two neighboring point or line spread functions overlap with the maximum of one over the first zero of the other.

In the case of point objects and a circular aperture, the dip in the middle of the two images is about $74 \%$ of the maximum.

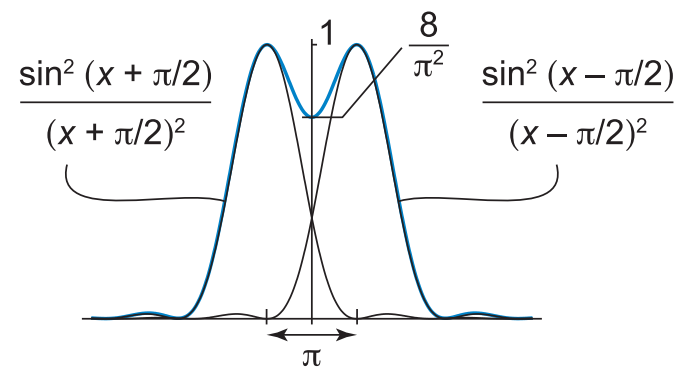

By the Rayleigh criterion, the angular resolution of a diffraction-limited telescope with a circular-entrance-pupil diameter $D$ and imaging wavelength $\lambda$ is

$$
\text { Angular resolution }=1.22 \lambda / D
$$

In spectroscopy, neighboring slit images must be resolved, so it is also common to apply the Rayleigh criterion to sinc functions as shown below.

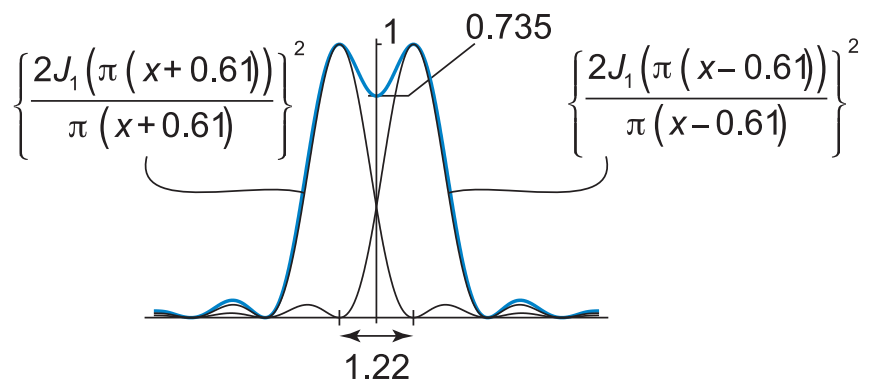




\section{Rotationally Symmetric Gaussian Beams}

The classical Gaussian beam closely approximates many real beams - primarily coherent laser beams-and is therefore of great importance in wave optics.

The basic form of the Gaussian beam, which is a solution to the paraxial Helmholtz equation, is a parabolic approximation to a spherical wave with complex radius curvature $q$ :

$$
U=u e^{i k z}=-\frac{i A}{q} e^{i \frac{k r^{2}}{2 q}} e^{i k z}
$$

The paraxial Helmholtz equation requires that $\partial q / \partial z=1$ or that $q=z+a+i z_{0}$, where $a$ and $z_{0}$ are real constants:

$$
u=-\frac{i A}{q} e^{i \frac{k r^{2}}{2|q|^{2}}\left(z+a+i z_{0}\right)}=-\frac{i A}{q} e^{i \frac{k r^{2}}{2|q|^{2}}(z+a)} e^{-\frac{k z z_{0} r^{2}}{2|q|^{2}}}
$$

It is customary to set $a=0$ so that the wavefront is flat at $z=0$. The Rayleigh range $z_{0}$-which must be positive so that $u$ has the form of a paraxial spherical wave with a Gaussian irradiance profile-is the distance between the maximum and minimum absolute curvature.

Imposing a form with a real radius of curvature $R$, standard deviation, $\sigma$, total power $\Phi$, and Gouy shift $\theta$,

$$
u=-\frac{i A}{q} e^{i \frac{k r^{2}}{2|q|^{2} / 2}} e^{\frac{k z_{0} r^{2}}{2|q|^{2}}}=\sqrt{\frac{\Phi}{\pi \sigma^{2}}} e^{i \frac{k r^{2}}{2 R}} e^{-\frac{r^{2}}{2 \sigma^{2}}} e^{-i \theta}
$$

where

$$
\begin{array}{ccc}
q=z-i z_{0} & R=z+z_{0}^{2} / z & A=k \sigma_{0} \sqrt{\Phi / \pi} \\
\sigma^{2}=\sigma_{0}^{2}\left(1+z^{2} / z_{0}^{2}\right) & z_{0}=k \sigma_{0}^{2} & \tan \theta=z / z_{0}
\end{array}
$$




\section{Rotationally Symmetric Gaussian Beams (cont.)}

Another common form of the Gaussian beam uses the $e^{-2}$ beam radius $w$.

$$
\begin{gathered}
u=-\frac{i A}{q} e^{-i \frac{k r^{2}}{2 q}}=\sqrt{\frac{2 \Phi}{\pi w^{2}}} e^{-i \frac{k r^{2}}{2 h}} e^{-\frac{r^{2}}{w^{2}}} e^{-i \theta} \\
w=w_{0} \sqrt{1+z^{2} / z_{0}^{2}} \\
R=-z-z_{0}^{2} / z \quad \tan \theta=z / z_{0} \quad \tan \delta=\frac{w_{0}}{z_{0}} \\
R=z+i z_{0} \quad z_{0}=\frac{1}{2} k w_{0}^{2}
\end{gathered}
$$

Independent of how the radius is defined (with $\sigma, w$, or something else), the radius of the beam traces out a hyperbola with propagation. The divergence of a Gaussian beam $\delta$, or sometimes $\tan \delta$, is the angle defined by the asymptote of the beam radius and happens to be the same angle defined by the beam-waist radius as viewed from the Rayleigh range.

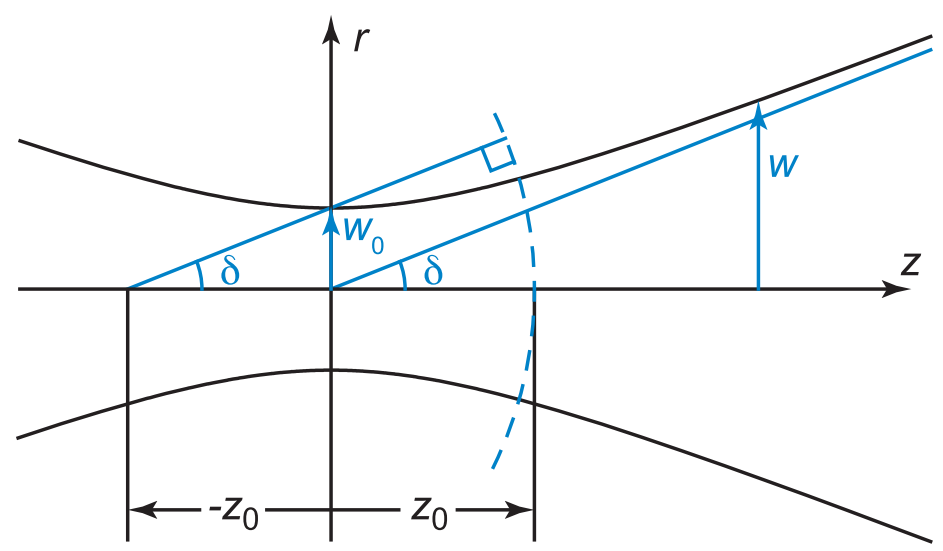




\section{Gaussian Beam Size}

The size of rotationally symmetric (or two-fold symmetric) Gaussian beams can be described in several ways. The beam size is usually given in terms of the irradiance, and the most common size parameters are the half-width at half-max $h$, the $1 / e$ radius $\sigma$, and the $1 / e^{2}$ radius $w$. These plots illustrate the relationship between these size parameters relative to the irradiance profile of the beam (top) and the beam divergence, which may also be defined using the same size parameters.

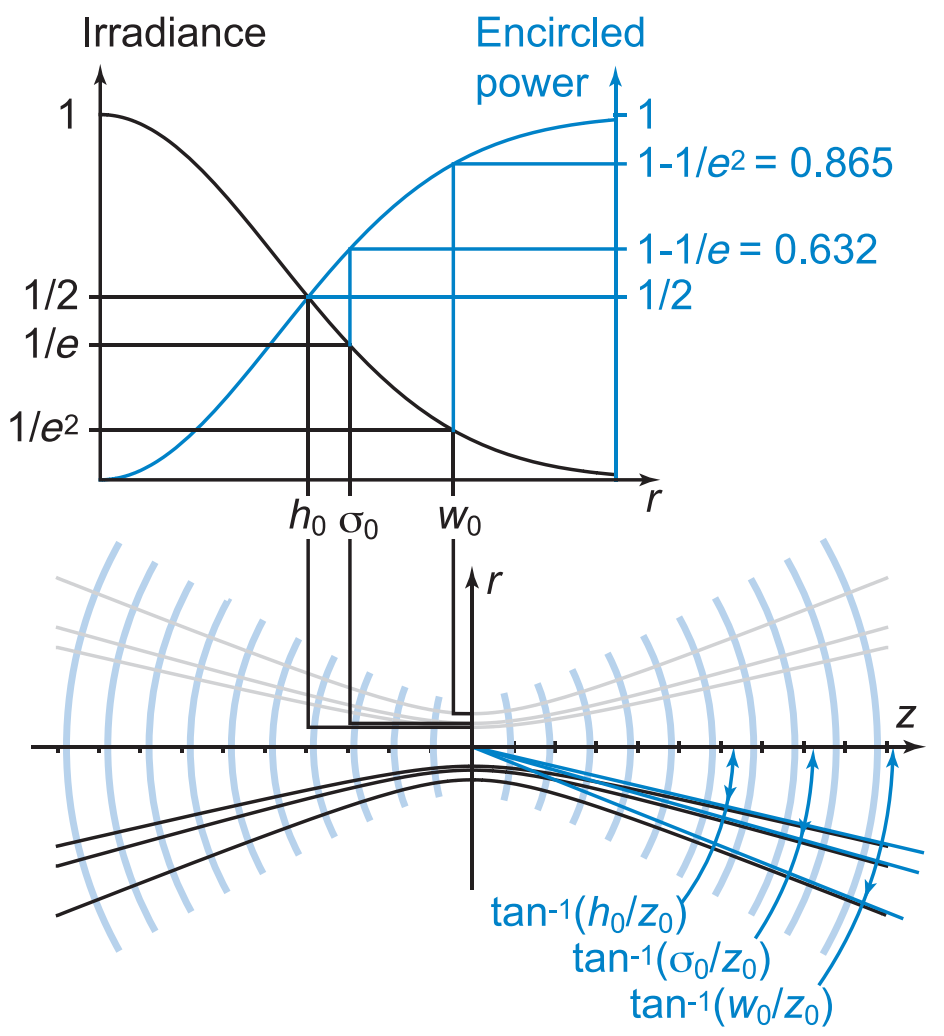

Although each of these parameters is a half-width, fullwidths are sometimes encountered as well. 


\section{Rayleigh Range and Sister Surfaces}

The Rayleigh range $z_{0}$ is perhaps best defined as the distance from the beam waist where the Gouy shift $\theta$ is $\pi / 4$ because this is true for general Gaussian beams, which are not necessarily rotationally symmetric and do not exhibit separability in $x$ and $y$.

In the case of a rotationally symmetric Gaussian beam, the Rayleigh range is simultaneously the distance from the waist where the wavefront curvature is maximum, where the beam area doubles, and where the axial irradiance halves. It also corresponds to a pair of sister surfaces positioned at equal distances on either side of the waist.

Sister surfaces are pairs of Gaussian beam wavefronts that are located at each other's center of curvature, as illustrated here.

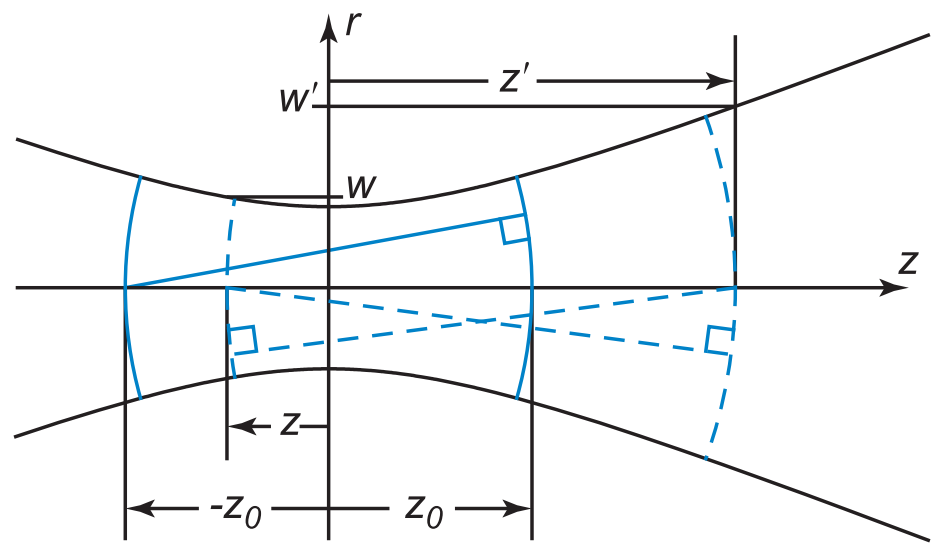

The positions, radii, and Gouy shifts of sister surfaces obey these relationships:

$$
z z^{\prime}=-z_{0}^{2}, \quad \frac{1}{w^{2}}+\frac{1}{w^{\prime 2}}=\frac{1}{w_{0}^{2}}, \quad\left|\theta-\theta^{\prime}\right|=\pi / 2
$$




\section{Gouy Shift and Wavefront Curvature}

The phase of a Gaussian beam relative to a parallelpropagating plane wave will lose $180 \mathrm{deg}$ as it goes from $-\infty$ to $+\infty$. This effect, which also exists in any focused beam, is known as the Gouy shift (often misspelled as "Guoy") and is captured in the Gaussian beam parameter $\theta$ :

$$
\tan \theta=\frac{z}{z_{0}}
$$

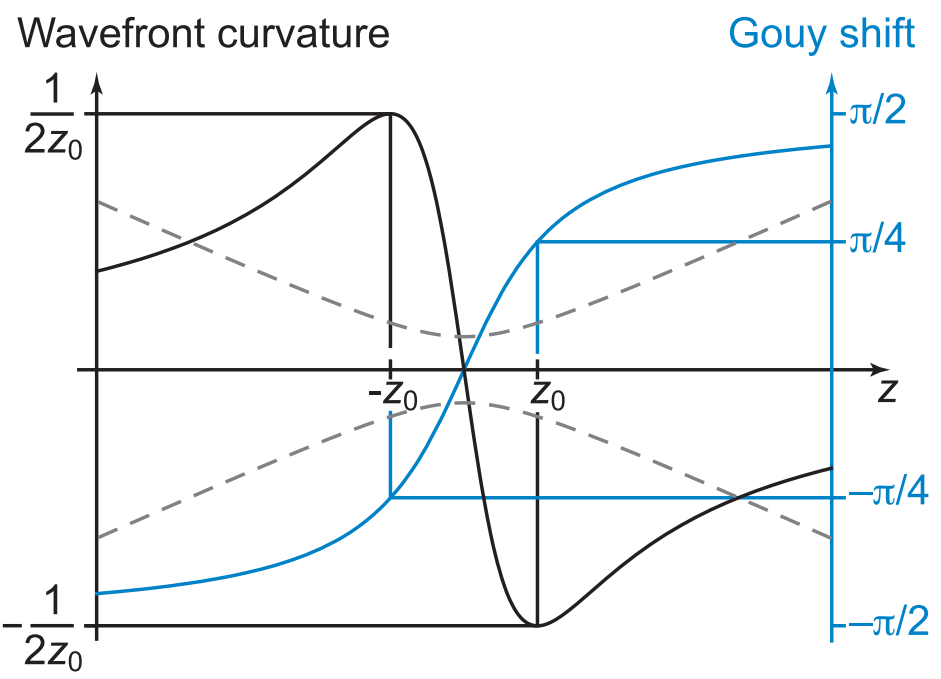

In this plot, the rotationally symmetric Gaussian beam profile is shown schematically with a dashed line superimposed on the plots of wavefront curvature and Gouy shift. It is clear that the Rayleigh range corresponds to the location of Gouy shift of $\pm \pi / 4$, as well as the positions of maximum absolute wavefront curvature (for the case of a rotationally symmetric Gaussian beam). 


\section{ABCD Method for Gaussian Beams}

The complex curvature $q$ completely specifies the unitpower rotationally symmetric Gaussian beam, and the ABCD method relates input and output complex curvatures $q$ and $q^{\prime}$ through the elements of a paraxial system matrix:

$$
q^{\prime}=\frac{A q+B}{C q+D}
$$

The system matrix (which can be formed from the submatrices shown in the table below) determines the propagation of a ray of initial height $y$ and the optical angle $\omega=n u$ (paraxial angle $u$ and refractive index $n$ ):

$$
\left(\begin{array}{l}
y^{\prime} \\
\omega^{\prime}
\end{array}\right)=\left(\begin{array}{ll}
A & B \\
C & D
\end{array}\right)\left(\begin{array}{l}
y \\
\omega
\end{array}\right)=\mathbf{M}_{n} \ldots \mathbf{M}_{2} \mathbf{M}_{1}\left(\begin{array}{l}
y \\
\omega
\end{array}\right)=\mathbf{M}\left(\begin{array}{l}
y \\
\omega
\end{array}\right)
$$

\begin{tabular}{|l|l|}
\hline $\begin{array}{l}\text { Transfer by reduced thickness } \\
\tau=t / n \text { (thickness } t \text { and index } n \text { ) }\end{array}$ & $\mathbf{M}_{T}=\left(\begin{array}{cc}1 & \tau \\
0 & 1\end{array}\right)$ \\
\hline Thin lens of power $\phi$ & $\mathbf{M}_{R}=\left(\begin{array}{cc}1 & 0 \\
-\phi & 1\end{array}\right)$ \\
\hline Conjugates at magnification $m$ & $\mathbf{M}_{C}=\left(\begin{array}{cc}m & 0 \\
-\phi & 1 / m\end{array}\right)$ \\
\hline Focal planes for lens of power $\phi$ & $\mathbf{M}_{F}=\left(\begin{array}{cc}0 & 1 / \phi \\
-\phi & 0\end{array}\right)$ \\
\hline $\begin{array}{l}\text { Nodal planes with initial and final } \\
\text { refractive indices } n \text { and } n^{\prime}\end{array}$ & $\mathbf{M}_{N}=\left(\begin{array}{cc}n / n^{\prime} & 1 / \phi \\
-\phi & n^{\prime} / n\end{array}\right)$ \\
\hline
\end{tabular}

Any system matrix can also be derived in reverse, given initial and final heights and optical directions of any two linearly independent rays:

$$
\mathbf{M}=\left(\begin{array}{ll}
A & B \\
C & D
\end{array}\right)=\left(\begin{array}{cc}
y_{1}^{\prime} & y_{2}^{\prime} \\
\omega_{1}^{\prime} & \omega_{2}^{\prime}
\end{array}\right)\left(\begin{array}{cc}
\omega_{1} & -y_{2} \\
-\omega_{1} & y_{2}
\end{array}\right) /\left|\begin{array}{ll}
y_{1} & y_{2} \\
\omega_{1} & \omega_{2}
\end{array}\right|
$$

